Research Article

\title{
Generalized Phenomenological Models of Dark Energy
}

\author{
Prasenjit Paul $\mathbb{D}^{1}$ and Rikpratik Sengupta $\mathbb{D}^{2}$ \\ ${ }^{1}$ Department of Physics, Indian Institute of Engineering Science and Technology, Shibpur, India \\ ${ }^{2}$ Department of Physics, Government College of Engineering and Ceramic Technology, Kolkata, 700010 West Bengal, India
}

Correspondence should be addressed to Prasenjit Paul; prasenjit071083@gmail.com

Received 30 July 2019; Accepted 28 November 2019; Published 20 February 2020

Academic Editor: Luca Stanco

Copyright (c) 2020 Prasenjit Paul and Rikpratik Sengupta. This is an open access article distributed under the Creative Commons Attribution License, which permits unrestricted use, distribution, and reproduction in any medium, provided the original work is properly cited. The publication of this article was funded by SCOAP ${ }^{3}$.

It was first observed at the end of the last century that the universe is presently accelerating. Ever since, there have been several attempts to explain this observation theoretically. There are two possible approaches. The more conventional one is to modify the matter part of the Einstein field equations, and the second one is to modify the geometry part. We shall consider two phenomenological models based on the former, more conventional approach within the context of general relativity. The phenomenological models in this paper consider a $\Lambda$ term firstly a function of $\ddot{a} / a$ and secondly a function of $\rho$, where $a$ and $\rho$ are the scale factor and matter energy density, respectively. Constraining the free parameters of the models with the latest observational data gives satisfactory values of parameters as considered by us initially. Without any field theoretic interpretation, we explain the recent observations with a dynamical cosmological constant.

\section{Introduction}

Type Ia high-redshift supernova observations indicate that the universe is presently accelerating $[1,2]$. This is mostly thought to be due to the presence of some unknown fluid known as dark energy. Soon after the first cosmological solution to the Einstein field equations (EFE), Einstein had put an additional $\Lambda$ term (known as the cosmological constant) which produced a repulsive effect, in order to modify the EFE so that the cosmological solution could lead to a static universe. He later called the introduction of this term to be the greatest blunder of his life. However, after observations suggested an accelerating universe, there was a revived interest in the $\Lambda$ term as a possible candidate for the dark energy. Theoretically, the cosmological constant is assumed to be the contribution from vacuum energy given by $\Lambda=8 \pi G \rho_{\mathrm{vac}}$, arising out of quantum vacuum fluctuations of some fundamental field. Although the calculated value of $\rho_{\mathrm{vac}}$ turns out to be much larger than the value of $\Lambda$ determined from observations, but there is no theoretical argument of making the $\rho_{\text {vac }}$ term vanish to exactly zero [3]. So $\Lambda$ models are favored for dark energy (DE). $\Lambda$ has also been thought to be generated from a particle creation effect or dynamical scalar field [4]. If we consider that the $\Lambda$ term is responsible for the dark energy, whatever is the generation mechanism, it is clear that contrary to Einstein, $\Lambda$ is not a constant but a dynamical cosmological term [5].

$\mathrm{DE}$ is also sometimes considered without the presence of any fluid or $\Lambda$ term, just as a consequence of the modification of the geometric part or the left hand side of the EFE, but such efforts are not possible in the context of standard general relativity (GR) $[6,7]$. There are also dynamically evolving scalar field models which have been used to describe DE. The popular dynamical physical field models that have been utilized for this purpose are quintessence [8-11], $k$-essence [12-17], phantom [18], and tachyonic field [19-26]. Phenomenological models of a dynamical $\Lambda$ term are also being popularly considered candidates of DE. "Phenomenological" simply means that there is no derivation of the dynamical $\Lambda$ term from any underlying quantum field theory. Such models may be categorized into three types: (i) kinematic, (ii) hydrodynamic, and (iii) field theoretic. The first means that $\Lambda$ is a function of time or scale factor $a(t)$. The second means that $\Lambda$ is treated as a barotropic fluid with 
some equation of state (EOS). The third means that $\Lambda$ is treated as a new physical classical field with phenomenological Lagrangian. We will be concerned here with (i) and (ii) only. Such kinematic and hydrodynamic models have been treated in some depth before. A dynamical model with $\Lambda=$ $\alpha H^{2}$, where $H(t)=\dot{a} / a$, has been explored by Mukhopadhyay et al. [27]. A similar model with $\dot{\Lambda} \sim H^{3}$ has been considered in $[28,29]$. The main motivation of considering $\Lambda \sim \ddot{a} / a$ and $\Lambda \sim \rho$ is to prove that these two dynamical models are equivalent for both open and closed universes in addition to the flat space, which has already been proved previously. The value of $\Lambda$ obtained theoretically from particle physics is several orders of magnitude greater than the observed value for $\Lambda$, if $\Lambda$ has to be considered dark energy. This is explained in various models as it is obtained that $\Lambda \sim 1 / t^{2}$ for flat spacetime and it is presently very small but nonzero, and this fact is well supported by our models.

The most frequently used forms of $\Lambda$ for phenomenological models are $\Lambda=\alpha(\dot{a} / a)^{2}, \Lambda=\beta(\ddot{a} / a)$, and $\Lambda=8 \pi G \gamma \rho$, where $\alpha, \beta$, and $\gamma$ are constants whose values can be constrained from observations. In general, the sign constraints on $\beta$ and $\gamma$ are imposed in order to ensure a positive value of the matter density parameter $\Omega_{m}$. The first type of model has been considered by [30-37]. The second model has been dealt with by $[5,38-40]$. The third type of model has been considered by [41]. The equivalence of these three forms has been shown by Ray et al. $[42,43]$, connecting the free parameters of the models with the matter density and vacuum energy density parameters in the first paper and by application of numerical methods in the later one. This paper is basically an in-depth extension of the work done by Mukhopadhyay et al. [44] where they have considered the first type of model and obtained cosmological solutions for any possible value of the curvature constant and equation of state parameter $\omega$. They have also analysed the physical features of the solutions. We shall do the same for the second and third models and also compare our results to the latest observational data constraining our free parameters. The constraints are found to be exactly compatible with our initial considerations.

The paper is organized as follows. In the second section, we consider the mathematical model in the background of an isotropic and homogeneous Friedmann-Lemaitre-Robertson-Walker (FLRW) space-time, which is the generally used cosmological metric in GR. We calculate the various cosmological and physical parameters for the two different phenomenological models in consideration. In the next section, we constrain the free parameters associated with the models based on recent observational data. The final section summarizes the physical insights of the results we have obtained.

\section{Mathematical Model}

The Einstein field equation (EFE) in the presence of a cosmological constant, $\Lambda(t)$, is given by

$$
G^{\mu v}=-8 \pi G\left[T^{\mu v}-\frac{\Lambda}{8 \pi G} g^{\mu v}\right],
$$

where we shall take the cosmological constant as a function of time in order to account for the dark energy. We obtain the EFE for the cosmological FLRW metric:

$$
d s^{2}=-d t^{2}+\left[\frac{d r^{2}}{1-k r^{2}}+r^{2}\left(d \theta^{2}+\sin ^{2} \theta d \phi^{2}\right)\right]
$$

which yields the equations

$$
\begin{aligned}
\left(\frac{\dot{a}}{a}\right)^{2}+\frac{k}{a^{2}} & =\frac{8 \pi G \rho}{3}+\frac{\Lambda}{3}, \\
\left(\frac{\ddot{a}}{a}\right) & =-\frac{4 \pi G(\rho+3 p)}{3}+\frac{\Lambda}{3},
\end{aligned}
$$

where $a(t)$ and $k$ are the scale factor and curvature constant, respectively.

The energy-momentum conservation gives

$$
8 \pi G(p+\rho)\left(\frac{\dot{a}}{a}\right)=-\frac{8 \pi G}{3} \dot{\rho}-\frac{\dot{\Lambda}}{3} .
$$

We consider the barotropic fluid with equation of state (EOS) of the form:

$$
p=\omega \rho,
$$

where $\omega$ denotes the EOS parameter which can assume specific values during the evolution of the universe for different phases. By plugging this relation in equation (4), the energy density is obtained as

$$
\rho=\frac{3}{4 \pi G(1+3 \omega)}\left(\frac{\Lambda}{3}-\frac{\ddot{a}}{a}\right)
$$

Substituting equation (6) into equation (4) multiplied by a factor of $2 /(1+3 \omega)$ and adding equation (3) to it, we get the differential equation

$$
\left(\frac{\dot{a}}{a}\right)^{2}+\frac{k}{a^{2}}+\frac{2}{1+3 \omega}\left(\frac{\ddot{a}}{a}\right)=\left(\frac{1+\omega}{1+3 \omega}\right) \Lambda .
$$

The above equation describes the cosmological dynamics for a barotropic fluid in the presence of the cosmological constant $\Lambda$.

2.1. Solutions for Phenomenological Model $\Lambda \sim \ddot{a} / a$. In this phenomenological model, we consider $\Lambda=\beta(\ddot{a} / a)$, where $\beta<0$ which is justified in the light of the latest observational data [45] as shown in Section 3. Using this form of $\Lambda$ in equation (8), we obtain

$$
\frac{\ddot{a}}{a}=-\frac{(1+3 \omega)}{2-(1+\omega) \beta} \frac{\dot{a}}{a}-\frac{(1+3 \omega) k}{2-(1+\omega) \beta} \frac{1}{a \dot{a}} .
$$




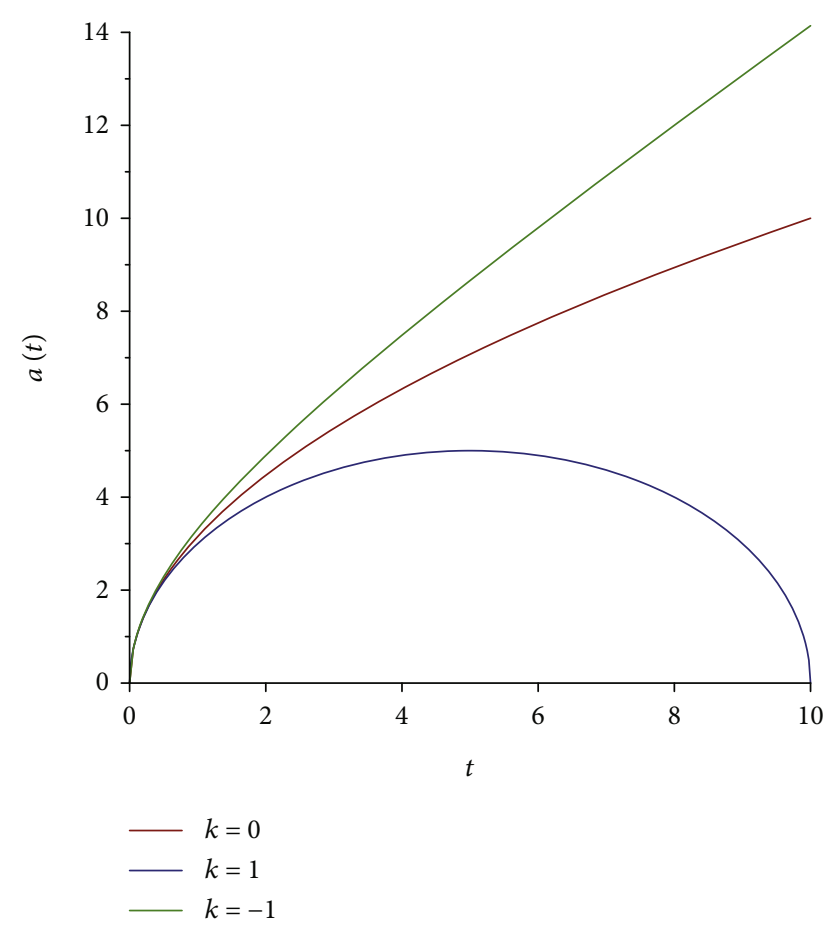

Figure 1: Plot of $a(t)$ versus $t$ for different values of $k$.

This equation can be simplified to

$$
a \dot{a} \frac{d}{d t}\left[\ln \left(\dot{a} a^{-A}\right)\right]=A k,
$$

where $A=-((1+3 \omega) /(2-(1+\omega) \beta))$. We choose $A=-1[?]$, such that $\omega=(1-\beta) /(3+\beta)$. Any arbitrary value can be taken. We take $A=-1$ and later $B=-1$ so that the differential equation can be solved analytically and complex numerical calculations may be avoided.

The above equation now takes the form

$$
a \dot{a} \frac{d}{d t}[\ln (\dot{a} a)]=-k
$$

The scale factor turns out to be

$$
a(t)=\sqrt{A_{0} t+A_{1}-k t^{2}},
$$

where $A_{0}$ and $A_{1}$ are integration constants.

As we are considering a universe evolving from a singularity, $a(t=0)=0$. This gives $A_{1}=0$. So

$$
a(t)=\sqrt{A_{0} t-k t^{2}} .
$$

We show the evolution of the scale factor with time for different values of $k$ in Figure 1. As expected, we obtain flat, open, and closed universes for $k=0, k=-1$, and $k=1$, respectively. The Hubble parameter is computed as

$$
H(t)=\frac{A_{0}-2 k t}{2\left(A_{0} t-k t^{2}\right)} .
$$

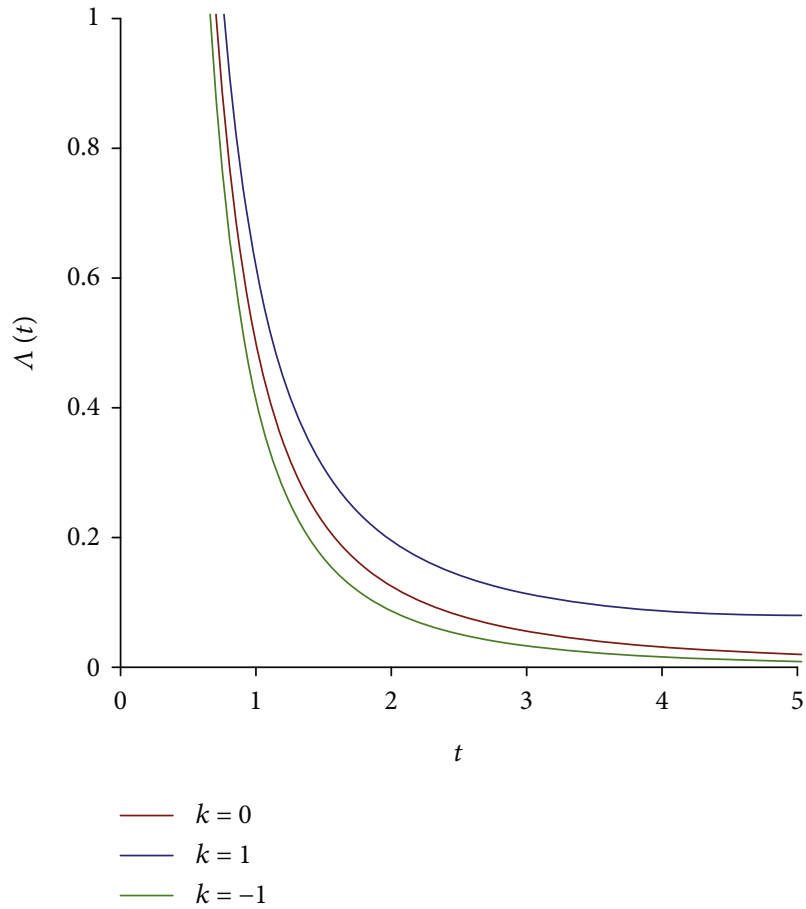

Figure 2: Plot of $\Lambda(t)$ versus $t$ for different values of $k$.

The cosmological constant is given by

$$
\Lambda(t)=-\frac{\beta A_{0}^{2}}{4\left(A_{0} t-k t^{2}\right)^{2}} .
$$

The variation of the cosmological parameter $\lambda$ with time is shown in Figure 2 and is found to be monotonically decreasing for all considered values of $k$. The energy density is given by

$$
\rho(t)=\frac{(3-\beta)}{16 \pi G} \frac{A_{0}^{2}}{\left(A_{0} t-k t^{2}\right)^{2}} .
$$

The variation of the scale factor and cosmological constant with time has been plotted for $k=0, \pm 1$ in Figures 1 and 2.

We obtain a closed universe for $k=1$ and an open universe for $k=-1$ as expected.

The density parameters for matter, a cosmological constant, and curvature, respectively, can be computed for this phenomenological model as

$$
\Omega_{m}=\frac{8 \pi G \rho}{3 H^{2}}=-\frac{4 \beta}{3}\left[\frac{k\left(A_{0} t-k t^{2}\right)}{\left(A_{0}-2 k t^{2}\right)^{2}}+\frac{1}{4}\right],
$$

$$
\Omega_{\Lambda}=\frac{\Lambda}{3 H^{2}}=\frac{4(3+\beta)}{3}\left[\frac{k\left(A_{0} t-k t^{2}\right)}{\left(A_{0}-2 k t^{2}\right)^{2}}+\frac{1}{4}\right],
$$




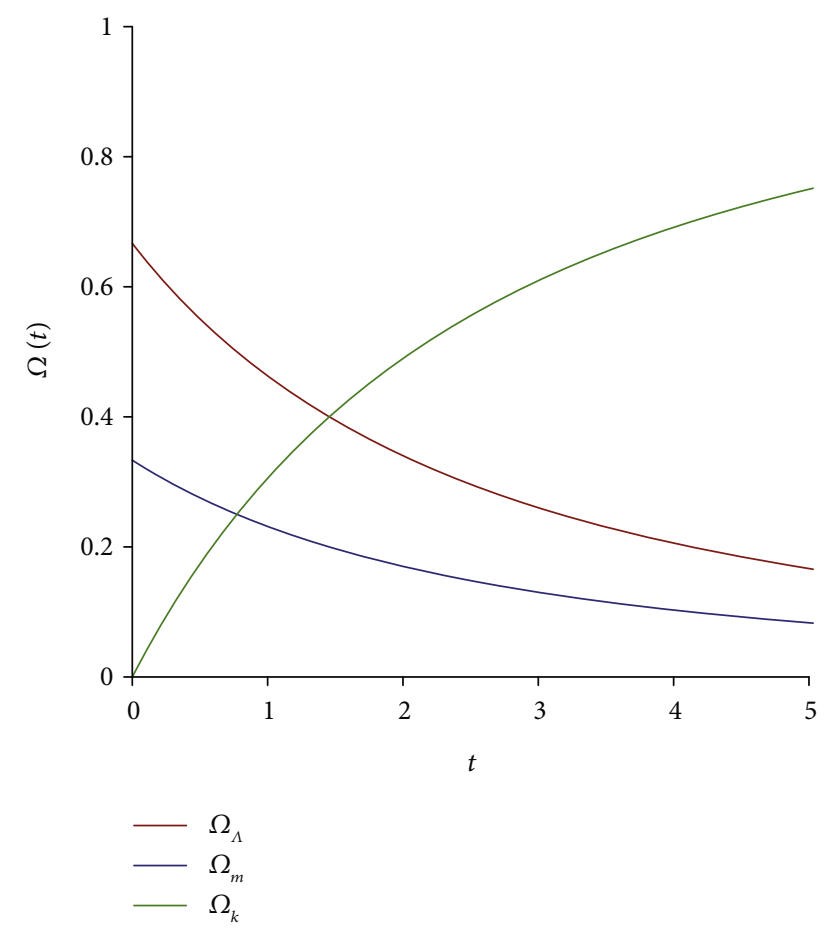

FIGURe 3: Plot of $\Omega(t)$ versus $t$ for $k=-1$.

$$
\Omega_{k}=-\frac{k}{a^{2} H^{2}}=-\frac{4 k\left(A_{0} t-k t^{2}\right)}{\left(A_{0}-2 k t^{2}\right)^{2}} .
$$

We plot the time evolution of the density parameters for all contributing components, i.e., matter, curvature, and dark energy, for $k=-1$ in Figure 3 . The contribution to the density parameter due to curvature increases while contributions due to $\Lambda$ and matter decrease, but the overall density parameter due to all three components remains the same.

We also plot the time evolution of the density parameters for matter, curvature, and dark energy for $k=1$ in Figure 4. The contribution to the density parameter due to curvature decreases and is negative while contributions due to $\Lambda$ and matter increase, but again the overall density parameter remains the same.

For the flat space $(k=0)$, we see from the above expressions that the sum total of the density parameters of the above components is equal to 1 , such that $\Omega_{k}=0, \Omega_{\Lambda}=$ $-(\beta / 3)$, and $\Omega_{m}=(3+\beta) / 3$.

Also, for both the limiting cases, $t \longrightarrow 0$ and $\infty$, the sum total of the density parameters is equal to 1 . In these two cases, both $\Omega_{m}$ and $\Omega_{\Lambda}$ become independent of $k$. Hence, for both early and late times, the universe exhibits similar behaviour as far as the $k$ dependency of $\Omega_{m}$ and $\Omega_{\Lambda}$ is concerned.

In general, it can be observed on taking the sum of equations (17)-(19) that

$$
\Omega_{m}+\Omega_{\Lambda}+\Omega_{k}=1 \text {. }
$$

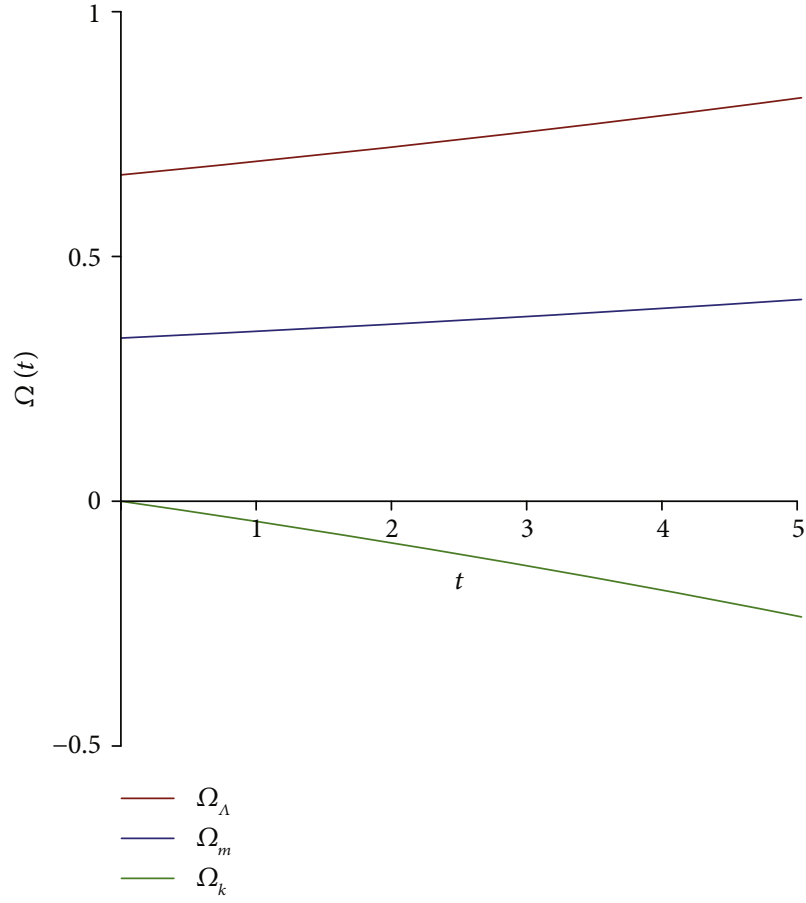

Figure 4: Plot of $\Omega(t)$ versus $t$ for $k=+1$.

This analytical approach is consistent with the observational constraints $\Omega=1 \pm 0.016$ [45].

2.2. Solutions for the Phenomenological Model $\Lambda \sim \rho$. In this phenomenological model, we consider $\Lambda=8 \pi G \rho \gamma$, where $\gamma>0$ which is consistent with the observation as can be seen in Section 3. Using this form of $\Lambda$ in equation (8), we obtain

$$
\frac{\ddot{a}}{\dot{a}}=-\frac{(1+3 \omega-2 \gamma)}{2(1+\gamma)} \frac{\dot{a}}{a}-\frac{(1+3 \omega-2 \gamma)}{2(1+\gamma)} \frac{k}{a \dot{a}} .
$$

This equation can be simplified to

$$
a \dot{a} \frac{d}{d t}\left[\ln \left(\dot{a} a^{-B}\right)\right]=B k
$$

where $B=(1+3 \omega-2 \gamma) / 2(1+\gamma)$. We choose $B=-1$, such that $\omega=(4 \gamma+1) / 3$.

The above equation now takes the form

$$
a \dot{a} \frac{d}{d t}[\ln (\dot{a} a)]=-k
$$

The scale factor turns out to be

$$
a(t)=\sqrt{A_{0}{ }^{\prime} t+A_{1}^{\prime}-k t^{2}}
$$

where $A_{0}{ }^{\prime}$ and $A_{1}{ }^{\prime}$ are integration constants. 


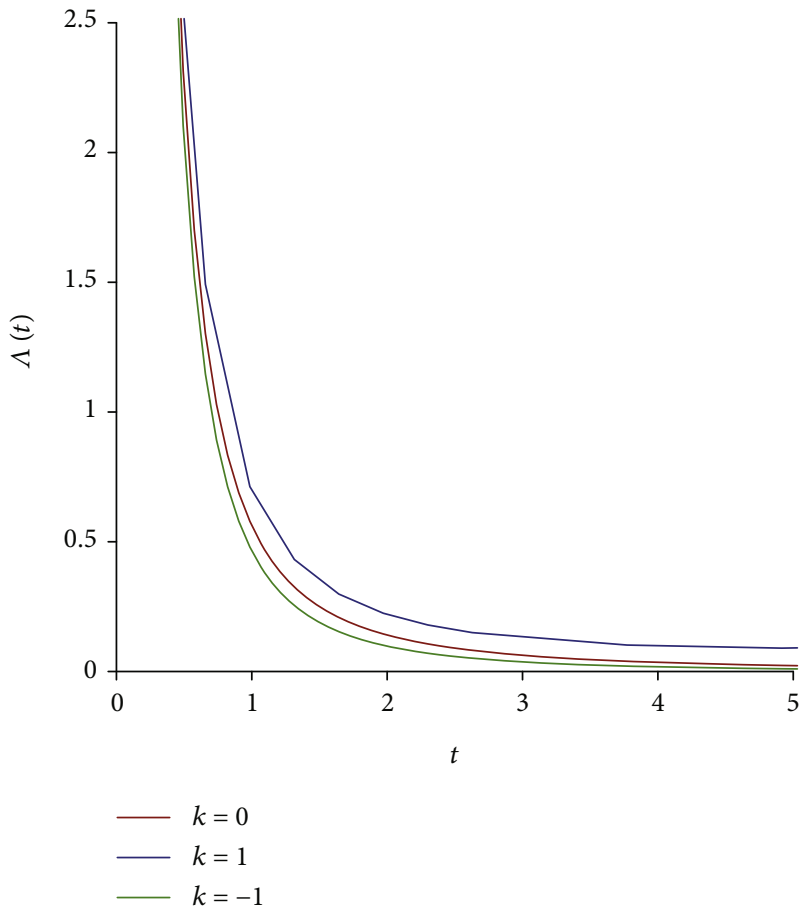

Figure 5: Plot of $\Lambda(t)$ versus $t$ for different values of $k$.

As we are considering a universe evolving from a singularity, $a(t=0)=0$. This gives $A_{1}^{\prime}=0$. So

$$
a(t)=\sqrt{A_{0}^{\prime} t-k t^{2}} .
$$

The Hubble parameter is computed as

$$
H(t)=\frac{A_{0}^{\prime}-2 k t}{2\left(A_{0}^{\prime} t-k t^{2}\right)} .
$$

The cosmological constant is given by

$$
\Lambda(t)=-\frac{3 \gamma A_{0}^{\prime 2}}{2(1+3 \omega-2 \gamma)\left(A_{0}^{\prime} t-k t^{2}\right)^{2}} .
$$

Time evolution of the cosmological constant is plotted in Figure 5 and is found to be monotonically decreasing for all considered values of $k$, similar to the $\beta$ model. The energy density is given by

$$
\rho(t)=\frac{3}{16 \pi G(1+3 \omega-2 \gamma)} \frac{A_{0}^{\prime 2}}{\left(A_{0}^{\prime} t-k t^{2}\right)^{2}} .
$$

Variation of the scale factor $a(t)$ is the same as shown in Figure 1.

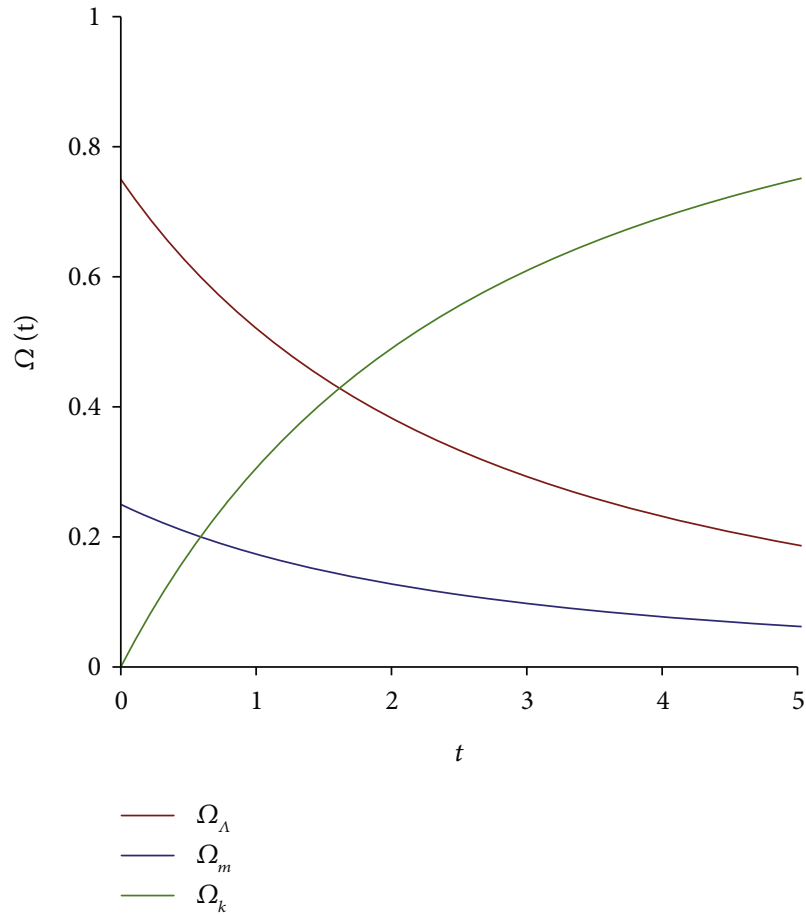

Figure 6: Plot of $\Omega(t)$ versus $t$ for $k=-1$.

The density parameters for matter, a cosmological constant, and curvature, respectively, can be computed in a similar manner as the above for this phenomenological model as

$$
\begin{aligned}
& \Omega_{m}=\frac{4 \gamma}{1+\gamma}\left[\frac{k\left(A_{0}^{\prime} t-k t^{2}\right)}{\left(A_{0}^{\prime}-2 k t^{2}\right)^{2}}+\frac{1}{4}\right], \\
& \Omega_{\Lambda}=\frac{4}{1+\gamma}\left[\frac{k\left(A_{0}^{\prime} t-k t^{2}\right)}{\left(A_{0}^{\prime}-2 k t^{2}\right)^{2}}+\frac{1}{4}\right], \\
& \Omega_{k}=-\frac{k}{a^{2} H^{2}}=-\frac{4 k\left(A_{0}^{\prime} t-k t^{2}\right)}{\left(A_{0}^{\prime}-2 k t^{2}\right)^{2}} .
\end{aligned}
$$

Similar to the previous model, we again plot the time evolution of the density parameters for all contributing components, i.e., matter, curvature, and dark energy, for $k=-1$ in Figure 6 . The contribution to the density parameter due to curvature increases while contributions due to $\Lambda$ and matter decrease, but the overall density parameter due to all three components remains the same; also, the time evolution of the density parameters for matter, curvature, and dark energy for $k=1$ has been plotted in Figure 7. Similarly, as for the previous model, the contribution to the density parameter due to curvature decreases and is negative while contributions due to $\Lambda$ and matter increase, but again the overall density parameter remains the same. 


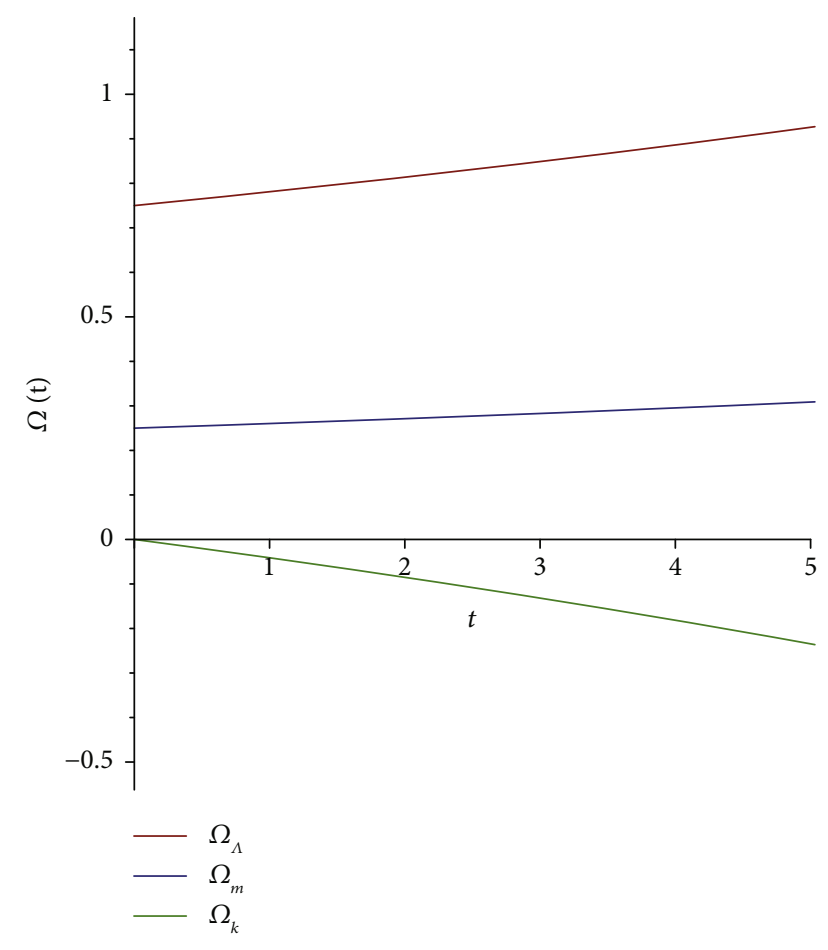

FiguRe 7: Plot of $\Omega(t)$ versus $t$ for $k=+1$.

For the flat space $(k=0)$, we see from the above expressions that the sum total of the density parameters of the above components is equal to 1 , such that $\Omega_{k}=0$, $\Omega_{m}=1 /(1+\gamma)$, and $\Omega_{\Lambda}=\gamma /(1+\gamma)$.

In the case of $t \rightarrow 0$ and $\infty$, the sum total of the density parameters is equal to 1 in a similar manner as for the previous model.

In general, it can be observed on taking the sum of the above three equations that

$$
\Omega_{m}+\Omega_{\Lambda}+\Omega_{k}=1
$$

which is again consistent with [45].

\section{Constraints on the Different Parameters with the Latest Observational Results}

Although we deal with simple phenomenological models which are not dependent on any quantum field theory, different cosmological pictures can be reflected successfully.

Considering the cosmology of base- $\Lambda$-CDM, late universe parameters can be observed in ranges: Hubble constant $H_{0}=67.4 \pm 0.5 \mathrm{~km} / \mathrm{s} / \mathrm{Mpc}$ and matter density parameter $\Omega_{m 0}=0.315 \pm 0.007$ [45]. Using the above ranges of $\Omega_{m 0}$, the model parameters can be constrained as $-2.076 \leq \beta_{0} \leq-2.034$ and $2.105 \leq \gamma_{0} \leq 2.247$. Using more recent data [46] where the present value of the Hubble parameter is given as $H_{0}=74.03 \pm 1.42 \mathrm{~km} / \mathrm{s} / \mathrm{Mpc}$, we obtain $-2.0717 \leq \beta_{0} \leq-2.06$ and $1.785 \leq \gamma_{0} \leq 1.821$.

The present value of the cosmological constant $\Lambda_{0}$ can be obtained using the relation $\Lambda_{0}=3 H_{0}^{2} \gamma_{0} \Omega_{m 0}$. It lies within the range $0.9 \times 10^{-35} \mathrm{~s}^{-2} \leq \Lambda_{0} \leq 1.042 \times 10^{-35} \mathrm{~s}^{-2}$, which is in sync with the observation [45]. We know the quintessence equation of state as $p_{Q}=\omega_{Q} \rho_{Q}, \omega_{Q}=-\Omega_{\Lambda}=-\gamma \Omega_{m}$. Using the above ranges we have $-0.724 \leq \omega_{Q} \leq-0.648$. This range is in good agreement with the accepted range of $\omega_{Q}$ which is $-1 \leq \omega_{Q} \leq-0.6[45,47,48]$, although, in either of our models, we do not consider quintessence and present the range only as a qualitative check.

Also, there are upcoming observational surveys like the Euclid survey [49] which is aimed at obtaining more precise and accurate data concerning dark energy and dark matter. The survey plans to use redshift selected baryon acoustic oscillation (BAO) to study dark energy and its time evolution. The phenomenological models which consider $\Lambda$ dark energy in the kinematic and hydrodynamic forms can also be constrained more stringently using precise data from this survey. This may decide the fate of these models in future study.

\section{Conclusion}

To summarize, the basic philosophy behind the present paper is to generalize two phenomenological models. Explicit expressions of $a(t), H(t), \rho(t), \Lambda(t)$, and also the parameter $\Omega(t)$ corresponding to matter, curvature, and DE have been derived. Cosmic evolution of the universe from the very early time to the late time has been discussed.

The conclusions of the present work can be jotted down as follows:

(i) The models $\Lambda \sim \ddot{a} / a$ and $\Lambda \sim \rho$ are equivalent for $k= \pm 1$

(ii) Both the models exhibit usual cosmological behaviour for early and late time universes. Initially chosen values of the model parameters are found to be in good agreement with the observational data

(iii) Constraints on the different cosmological variables have been evaluated using our models, and the results are in good agreement with the observational results

\section{Data Availability}

We have not generated any data in this paper but used observational data from references [45-48].

\section{Conflicts of Interest}

The authors declare that they have no conflicts of interest.

\section{References}

[1] S. Perlmutter, G. Aldering, G. Goldhaber et al., "Measurements of $\Omega$ and $\Lambda$ from 42 high-redshift supernovae," The Astrophysical Journal, vol. 517, no. 2, pp. 565-586, 1999.

[2] A. G. Riess, R. P. Kirshner, B. P. Schmidt et al., "BVRI light curves for 22 type Ia supernovae," The Astrophysical Journal, vol. 117, 1999. 
[3] A. Aguirre, "Intergalactic dust and observations of type Ia supernovae," The Astrophysical Journal, vol. 525, no. 2, pp. 583-593, 1999.

[4] V. Sahni and A. A. Starobinsky, "The case for a positive cosmological $\Lambda$-term," International Journal of Modern Physics D: Gravitation; Astrophysics and Cosmology, vol. 9, no. 4, pp. 373-443, 2000.

[5] J. M. Overduin and F. I. Cooperstock, "Evolution of the scale factor with a variable cosmological term," Physical Review D, vol. 58 , no. $4,1998$.

[6] A. Lue, "The phenomenology of Dvali-Gabadadze-Porrati cosmologies," Physics Reports, vol. 423, no. 1, pp. 1-48, 2006.

[7] S. Nojiri and S. D. Odintsov, "Introduction to modified gravity and gravitational alternative for dark energy," International Journal of Geometric Methods in Modern Physics, vol. 4, no. 1, pp. 115-145, 2007.

[8] I. Zlatev, L. Wang, and P. J. Steinhardt, "Quintessence, cosmic coincidence, and the cosmological constant," Physical Review Letters, vol. 82, no. 5, pp. 896-899, 1999.

[9] P. Brax and J. Martin, "Robustness of quintessencePhysical Review D," vol. 61, no. 10, 2000.

[10] T. Barreiro, E. J. Copeland, and N. A. Nunes, "Quintessence arising from exponential potentials," Physical Review D, vol. 61 , no. 12, 2000.

[11] A. Albrecht and C. Skordis, "Phenomenology of a realistic accelerating universe using only Planck-scale physics," Physical Review Letters, vol. 84, no. 10, pp. 2076-2079, 2000.

[12] C. Armendáriz-Picón, T. Damour, and V. Mukhanov, " $k$ Inflation," Physics Letters B, vol. 458, no. 2-3, pp. 209-218, 1999.

[13] J. Garriga and V. F. Mukhanov, "Perturbations in $k$-inflation," Physics Letters B, vol. 458, no. 2-3, pp. 219-225, 1999.

[14] T. Chiba, T. Okabe, and M. Yamaguchi, "Kinetically driven quintessence," Physical Review D, vol. 62, no. 2, 2000.

[15] C. Armendariz-Picon, V. Mukhanov, and P. J. Steinhardt, "Dynamical solution to the problem of a small cosmological constant and late-time cosmic acceleration," Physical Review Letters, vol. 85, no. 21, pp. 4438-4441, 2000.

[16] C. Armendariz-Picon, V. Mukhanov, and P. J. Steinhardt, "Essentials of $k$-essence," Physical Review D, vol. 63, no. 10, p. 103510, 2001.

[17] M. Malquarti and A. R. Liddle, "Initial conditions for quintessence after inflation," Physical Review D, vol. 66, no. 2, 2002.

[18] R. R. Caldwell, "A phantom menace? Cosmological consequences of a dark energy component with super-negative equation of state," Physics Letters B, vol. 545, no. 1-2, pp. 23$29,2002$.

[19] A. Sen, "Rolling tachyon," Journal of High Energy Physics, vol. 2002, no. 4, 2002.

[20] A. Sen, "Tachyon matter," Journal of High Energy Physics, vol. 2002, no. 7, 2002.

[21] M. R. Garousi, "Tachyon couplings on non-BPS D-branes and Dirac-Born-Infeld action," Nuclear Physics B, vol. 584, no. 1-2, pp. 284-299, 2000.

[22] M. R. Garousi, "On-shell S-matrix and tachyonic effective actions," Nuclear Physics B, vol. 647, no. 1-2, pp. 117-130, 2002.

[23] M. R. Garousi, "Slowly varying tachyon and tachyon potential," Journal of High Energy Physics, vol. 2003, no. 5, p. 058, 2003.
[24] E. A. Bergshoeff, M. de Roo, T. C. de Wit, E. Eyras, and S. Panda, "T-duality and actions for non-BPS D-branes," Journal of High Energy Physics, vol. 5, 2000.

[25] J. Kluson, "Proposal for non-Bogomol'nyi-Prasad-Sommerfield D-brane action," Physical Review D, vol. 62, no. 12, p. 126003, 2000.

[26] D. Kutasov and V. Niarchos, "Tachyon effective actions in open string theory," Nuclear Physics B, vol. 666, no. 1-2, pp. 56-70, 2003.

[27] U. Mukhopadhyay, S. Ray, A. A. Usmani, and P. P. Ghosh, "Time variable $\Lambda$ and the accelerating universe," International Journal of Theoretical Physics, vol. 50, no. 3, pp. 752-759, 2011.

[28] S. Ray, F. Rahaman, U. Mukhopadhyay, and R. Sarkar, "Variable equation of state for generalized dark energy model," International Journal of Theoretical Physics, vol. 50, no. 9, pp. 2687-2696, 2011.

[29] A. A. Usmani, P. P. Ghosh, U. Mukhopadhyay, P. C. Ray, and S. Ray, "The dark energy equation of state," Monthly Notices of the Royal Astronomical Society: Letters, vol. 386, no. 1, pp. L92-L95, 2008.

[30] J. C. Carvalho, J. A. S. Lima, and I. Waga, "Cosmological consequences of a time-dependent $\Lambda$ term," Physical Review D, vol. 46, no. 6, pp. 2404-2407, 1992.

[31] I. Waga, "Decaying vacuum flat cosmological modelsexpressions for some observable quantities and their properties," The Astrophysical Journal, vol. 414, p. 436, 1993.

[32] J. A. S. Lima and J. C. Carvalho, "Dirac's cosmology with varying cosmological constant," General Relativity and Gravitation, vol. 26, no. 9, pp. 909-916, 1994.

[33] J. M. Salim and I. Waga, "Thermodynamic constraints on a time-dependent lambda model (cosmology)," Classical and Quantum Gravity, vol. 10, no. 9, pp. 1767-1774, 1993.

[34] A. I. Arbab and A.-M. M. Abdel-Rahman, "Nonsingular cosmology with a time-dependent cosmological term," Physical Review D, vol. 50, no. 12, pp. 7725-7728, 1994.

[35] C. Wetterich, "An asymptotically vanishing time-dependent cosmological constant," Astronomy and Astrophysics, vol. 301, p. 321, 1995.

[36] A. I. Arbab, "Cosmological models with variable cosmological and gravitational "constants" and bulk viscous models," General Relativity and Gravitation, vol. 29, no. 1, pp. 61-74, 1997.

[37] T. Padmanabhan, https://arxiv.org/abs/1907.12869v1.

[38] A. I. Arbab, "Cosmic acceleration with a positive cosmological constant," Classical and Quantum Gravity, vol. 20, no. 1, pp. 93-99, 2003.

[39] A. I. Arbab, "Cosmological consequences of a built-in cosmological constant model," Journal of Cosmology and Astroparticle Physics, vol. 5, p. 008, 2003.

[40] A. I. Arbab, "The equivalence between different dark (matter) energy scenarios," Astrophysics and Space Science, vol. 291, no. 2, pp. 143-149, 2004.

[41] R. G. Vishwakarma, "Consequences on variable $\Lambda$-models from distant type Ia supernovae and compact radio sources," Classical and Quantum Gravity, vol. 18, no. 7, pp. 1159-1172, 2001.

[42] S. Ray, P. C. Ray, M. Khlopov, P. P. Ghosh, U. Mukhopadhyay, and P. Chowdhury, "Scenario of inflationary cosmology from the phenomenological $\Lambda$ models," International Journal of Theoretical Physics, vol. 48, no. 9, pp. 2499-2510, 2009.

[43] S. Ray, U. Mukhopadhyay, and X.-H. Meng, "Accelerating universe with a dynamic cosmological term," Gravitation and cosmology, vol. 13, p. 142, 2007. 
[44] U. Mukhopadhyay, P. C. Ray, S. Ray, and S. B. Datta Choudhury, "Generalized model for $\Lambda$ - dark energy," International Journal of Modern Physics D: Gravitation; Astrophysics and Cosmology, vol. 18, p. 389, 2009.

[45] N. Aghanim et al., Planck Collaboration, https://arxiv.org/abs/ 1807.06209.

[46] A. G. Riess, S. Casertano, W. Yuan, L. M. Macri, and D. Scolnic, "Large Magellanic Cloud Cepheid standards provide a $1 \%$ foundation for the determination of the Hubble constant and stronger evidence for physics beyond $\Lambda \mathrm{CDM}$," The Astrophysical Journal, vol. 876, no. 1, p. 85, 2019.

[47] A. Balbi, C. Baccigalupi, S. Matarrese, F. Perrotta, and N. Vittorio, "Implications for quintessence models from MAXIMA-1 and BOOMERANG-98," The Astrophysical Journal, vol. 547, no. 2, pp. L89-L92, 2001.

[48] P. S. Corasaniti and E. J. Copeland, "Constraining the quintessence equation of state with SnIa data and CMB peaks," Physical Review D, vol. 65, no. 4, 2002.

[49] R. Laureijs et al., https://arxiv.org/abs/1110.3193. 